\title{
PATTERNS AND FACTORS AFFECTING WIVES' INFLUENCE IN FAMILY PURCHASE DECISION-MAKING IN URBAN MALAYSIA
}

\author{
SAMSINAR MD SIDIN \\ DAHLIA ZAWAWI \\ Faculty of Economics and Management \\ Universiti Putra Malaysia \\ CAROL TEO BOON HUI \\ Faculty of Business Management \\ Universiti Teknologi MARA
}

\begin{abstract}
Family purchase decision-making is the process by which decisions regarding purchases for the families are made. The roles played by family members differ with regard to the products being purchased, the stage in the decisionmaking process, and the characteristics of families and spouses. This study aims to investigate the family purchase decision-making process in urban Malaysia and the factors affecting this process. Taking into consideration past research, four hypotheses were developed. These hypothese were based on products / services, stage of the decision-making process, and selected demographic variables (income, occupation, education). A survey using a structured questionnaire was used to collect data $(N=1,000)$ in four different regions in Malaysia (Klang Valley, Penang, Kuantan, Johor Bahru). The findings of this study revealed that the majority of the products/services purchased for the family was a joint decision. The wives' influence generally decreased in the outcome stage, i.e., when products were actually purchased. The analyses of the effects of the selected demographic variables revealed that generally, there were significant effects of these variables on the majority of the purchases.
\end{abstract}

Keywords: Decision-making, demographic variables, family purchases, role structure, wives' influence.

\section{Introduction}

Family is defined as a group of people who are related by blood, marriage or adoption and live together. It is an important consumer unit as most purchases are bought by families or individuals for the 
consumption of the family. Purchase decisions by families are unique and complex as the decision-making process involves more than one person. One's spouse and children will have a strong direct influence on the purchase behaviour. The roles of the husbands and the wives are the focus of research in family decision-making as they are the most basic unit in the family (Schiffman \& Kanuk, 2010). Marketing researchers are interested to study family decision-making as information regarding the process, and input of the decision-making process is important in predicting consumer intention and purchase. This study aims to investigate the family purchase decision-making process in urban Malaysia and the factors affecting this process. As the effects of modernization have brought about changes in family structure in Malaysia, this study would be able to highlight the effects of these changes on family purchase decision-making.

The institution of family has changed over time. There are many changes in perceptions and roles within the family due to industrializationcaused social changes. More education and working opportunities are available to women, creating a different perception of the role of women and children in the family, and the role of husbands as the head of the family. It is also apparent that a wife's power in the family increases when the wife is employed outside of the house, a situation that is brought about by the societal development of the country. Social and economic developments have also brought changes in cultural values which consequently affect the role structure in family purchase decision-making.

\section{Family Purchase Decision-Making}

Family purchase decision-making is the process by which decisions regarding purchases for the families are made. Most purchases by the family will affect the family members directly, as both the process and the outcomes will affect the well-being of family members and the family as a unit (Hawkins, 2004). Family purchase decision-making involves different stages, depending on the product being purchased. These stages are the initial information gathering and evaluation stages and the outcome stages. The roles played by family members differ with regard to the products being purchased, the stage in the decision-making process, and the characteristics of families and spouses (Samsinar \& Rao, 2005). These roles may change over time due to changes in the environment, such as economic development, which consequently may lead to adjustments in the role structure of the decision-making process. The framework for the decision-making process relevant for this study can be seen in Figure 1. 


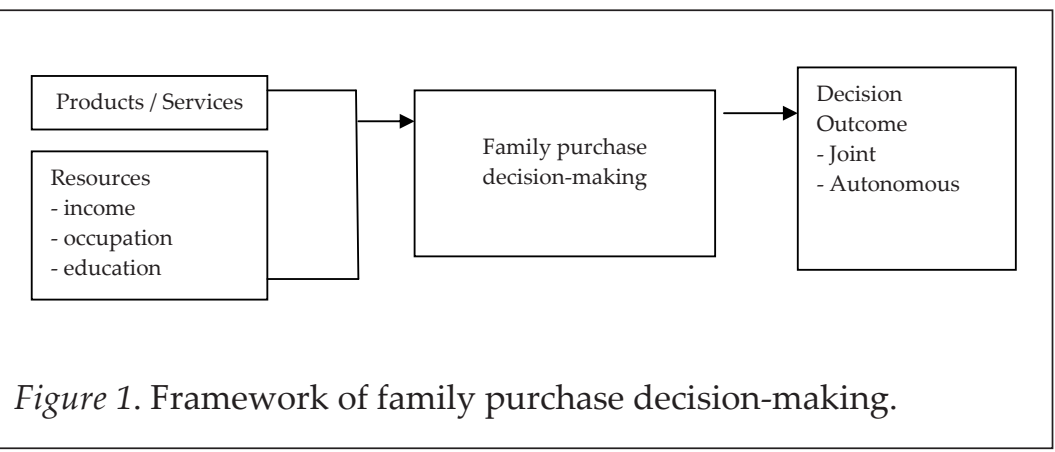

One major determinant of who has the final decision-making authority in the family is determining who controls the most resources. In this respect, resources such as income, education, and occupation are surrogate currencies used for negotiation in a family's decisionmaking. These surrogate currency is used to bargain for the desired goals in decision-making (Samsinar, Wong, Dahlia, Ruhana \& Zalfa Laili, 2004). This theory, referred to as resource theory, suggests that power in a family is determined by the ability of each spouse to provide for the needs of the family. Taking into consideration past research, four hypotheses were developed to test whether wives' influence varies with the following variables:

(i) products / services,

(ii) wives' income,

(iii) wives' occupation and

(iv) wives' education.

\section{Method}

A survey using a structured questionnaire was used to collect data. One thousand $(1,000)$ respondents were included in the study. The sample elements were selected based on race $(60 \%$ Malays, $30 \%$ Chinese and $10 \%$ Indians), occupation (50\% working wives, $50 \%$ housewives) and location (25\% from each different region).

Family is operationalised as a man and a woman married and living together for at least a year. Family purchase decision-making is operationalised as the process that a family goes through in the purchase of products used by the whole family. Fifteen products and services were used in the study. These products had been used in previous studies (Xia, Ahmed, Hwa, Tan \& Teo, 2006) and 
included furniture, electrical appliances, clothing, vacation, and cars. A modified measure used by previous researchers (Xia et al., 2006) to measure wives' influence was used. The means of the influence were calculated for each product/service category; accordingly, mean scores between 1-1.7 were considered as husband-dominant, mean scores between 1.71-2.3 were perceived as joint decisions and mean scores between 2.31-3 were considered as wife-dominant. Wives' resources were measured using the wives' occupations, incomes and education. These measures were used previously in similar studies in Malaysia (Samsinar \& Mary, 1996). These measures were tested for reliability and validity and were found to be both valid and reliable. Data was analysed using descriptive as well as statistical analysis. Analysis of variance, Chi-square analysis and t-tests were conducted accodingly consistent with the objectives of this study.

\section{Profile of Respondents}

The profile of the respondents is given in Table 1 and it can be seen that with respect to age the 31-40 years age group made up the largest group of respondents with $33.3 \%$, followed by the 21-30 years group with $28.5 \%$. Overall, about $86 \%$ of the respondents were between the ages $21-50$ years. In terms of race, Malays represented $57.3 \%$, Chinese represented $30.4 \%$ and Indians represented $11.8 \%$ of the respondents respectively. Muslims respondents (58.4\%) represented twice as much as Buddhists (23.4\%), whilst Christians (8\%) and Hindus (8.8\%) represented almost equal percentages of the respondents. Those of other religions made up $1.4 \%$ of the respondents.

In terms of occupation, housewives made up the largest block of respondents $(43.3 \%)$, followed by clerical $(15.6 \%)$, professional $(12.8 \%)$, and management $(12 \%)$. With regards to the duration of employment, those who had worked 10 years or less made up $72.2 \%$ of the respondents.

As for monthly income, those who made between RM1001-RM5000 formed the largest respondent group $(41.9 \%)$ and they were closely followed by the no-income group (39\%). In addition, those who earned RM5000 or less made up about $90 \%$ of the respondents.

In terms of educational level, $41.7 \%$ of the respondents had SPM or its equivalent and $21 \%$ had diploma or equivalent qualifications. Masters or $\mathrm{PhD}$ holders represented only $3 \%$ of the respondents. About $23 \%$ 
of the respondents had been married for 5 years or less and $63 \%$ for 15 years or less. Respondents who had been married for between 21-25 years made up the least group (9.2\%). The dominant language spoken at home was Malay (55.6\%), followed by Chinese $(22.2 \%)$ and English (12.8\%), whilst Tamil was spoken by $7.8 \%$ of the respondents.

\section{Table 1}

\section{Profile of Respondents}

\begin{tabular}{|c|c|c|c|c|}
\hline & & Frequency & Per cent & $\begin{array}{c}\text { Cumulative } \\
\text { per cent }\end{array}$ \\
\hline \multirow[t]{5}{*}{ Age } & $21-30$ years & 285 & 28.5 & 28.5 \\
\hline & $31-40$ years & 333 & 33.3 & 61.9 \\
\hline & $41-50$ years & 239 & 23.9 & 85.8 \\
\hline & $51-60$ years & 135 & 13.5 & 99.3 \\
\hline & More than 60 years & 7 & 0.7 & 100.0 \\
\hline \multirow[t]{4}{*}{ Race } & Malay & 573 & 57.3 & 57.4 \\
\hline & Chinese & 304 & 30.4 & 87.8 \\
\hline & Indian & 118 & 11.8 & 99.6 \\
\hline & Others & 4 & 0.4 & 100.0 \\
\hline \multirow[t]{5}{*}{ Religion } & Islam & 584 & 58.4 & 58.4 \\
\hline & Buddhism & 234 & 23.4 & 81.8 \\
\hline & Christianity & 80 & 8.0 & 89.8 \\
\hline & Hinduism & 88 & 8.8 & 98.6 \\
\hline & Others & 14 & 1.4 & 100.0 \\
\hline \multirow[t]{8}{*}{ Occupation } & Management & 120 & 12.0 & 12.0 \\
\hline & Professional & 128 & 12.8 & 24.8 \\
\hline & Clerical & 156 & 15.6 & 40.4 \\
\hline & Entrepreneur & 53 & 5.3 & 45.7 \\
\hline & Retired & 21 & 2.1 & 47.8 \\
\hline & Housewife & 433 & 43.3 & 91.2 \\
\hline & Student & 7 & 0.7 & 91.9 \\
\hline & Others & 81 & 8.1 & 100.0 \\
\hline \multirow{7}{*}{$\begin{array}{c}\text { Job } \\
\text { duration }\end{array}$} & Not working & 410 & 41.0 & 41.2 \\
\hline & Less than 5 years & 170 & 17.0 & 58.2 \\
\hline & $6-10$ years & 139 & 13.9 & 72.2 \\
\hline & $11-15$ years & 116 & 11.6 & 83.8 \\
\hline & $16-20$ years & 72 & 7.2 & 91.1 \\
\hline & $21-25$ years & 48 & 4.8 & 95.9 \\
\hline & More than 25 years & 41 & 4.1 & 100.0 \\
\hline
\end{tabular}


IJMS 19 (2), 47-73 (2012)

\begin{tabular}{clrrr}
\hline & & Frequency & Per cent & $\begin{array}{c}\text { Cumulative } \\
\text { per cent }\end{array}$ \\
\hline Monthly & No income & 390 & 39.0 & 39.0 \\
& Less than RM1k & 87 & 8.7 & 47.7 \\
& RM1001-RM5000 & 419 & 41.9 & 89.6 \\
& RM5001-RM10000 & 87 & 8.7 & 98.3 \\
& RM10001-RM15000 & 13 & 1.3 & 99.6 \\
& More than RM20000 & 4 & 0.4 & 100.0 \\
Education & SPM or equivalent & 417 & 41.7 & 41.9 \\
& STPM or equivalent & 99 & 9.9 & 51.9 \\
& Diploma or equivalent & 210 & 21.0 & 73.0 \\
& Bachelor degree or equivalent & 171 & 17.1 & 90.2 \\
& Masters or PhD & 30 & 3.0 & 93.2 \\
& Others & 68 & 6.8 & 100.0 \\
Marriage & Less than 5 years & 231 & 23.1 & 23.1 \\
& 6-10 years & 199 & 19.9 & 43.1 \\
& 11-15 years & 199 & 19.9 & 63.0 \\
16-20 years & 149 & 14.9 & 78.0 \\
21-25 years & 92 & 9.2 & 87.2 \\
& More than 25 years & 128 & 12.8 & 100.0 \\
Malay & 556 & 55.6 & 56.5 \\
& Chinese & 222 & 22.2 & 79.1 \\
& Tamil & 7.8 & 87.0 \\
& English & 12.8 & 100.0 \\
\hline
\end{tabular}

\section{Descriptive Statistics}

The mean influence values for the products/services across the three different stages of the decision- making process is shown in Table 2. From the table it can be seen that for computers $(\mathrm{M}=$ $1.63)$ and cars $(M=1.62)$, the buying decision is the husband's at all levels of the decision-making process. For homes and insurance it is the husband's decision at only the outcome stage but a jointdecision for all the other stages. The table also shows that it is the wife's decision for her clothes $(\mathrm{M}=2.54)$ at all stages of the decision-making process. Also for groceries $(\mathrm{M}=2.40)$ and children's clothes $(M=2.39)$ it is the wife's decision even though it is a joint decision at the outcome stage of the decision-making process for both products. For all other products/services the buying decision is basically a joint decision. 
IJMS 19 (2), 47-73 (2012)

Table 2

Mean Values for Products/Services at Each Decision Stage

\begin{tabular}{llcccc}
\hline Products/Services & Initial stage & $\begin{array}{c}\text { Evaluation } \\
\text { stage }\end{array}$ & $\begin{array}{c}\text { Outcome } \\
\text { stage }\end{array}$ & Mean \\
\hline 1 & Furniture & 2.22 & 2.06 & 1.84 & 2.04 \\
2 & Electrical appliances & 1.95 & 1.91 & 1.69 & 1.85 \\
3 & Computers & 1.65 & 1.67 & 1.58 & 1.63 \\
4 & Groceries & 2.56 & 2.41 & 2.23 & 2.40 \\
5 & Children's clothes & 2.51 & 2.40 & 2.26 & 2.39 \\
6 & Wife's clothes & 2.64 & 2.54 & 2.43 & 2.54 \\
7 & Husband's clothes & 1.83 & 1.87 & 1.75 & 1.82 \\
8 & Vacation & 1.93 & 1.91 & 1.78 & 1.87 \\
9 & Eating out & 1.93 & 1.89 & 1.75 & 1.86 \\
10 & Education & 2.01 & 1.97 & 1.88 & 1.95 \\
11 & Entertainment & 1.95 & 1.97 & 1.83 & 1.92 \\
12 & Bank account & 1.91 & 1.88 & 1.79 & 1.86 \\
13 & Insurance & 1.74 & 1.74 & 1.61 & 1.70 \\
14 & Home (buy/rent) & 1.75 & 1.76 & 1.60 & 1.70 \\
15 & Cars & 1.64 & 1.65 & 1.56 & 1.62 \\
& Overall mean & 2.01 & 1.98 & 1.84 & 1.94 \\
\hline
\end{tabular}

\section{Hypotheses Testing}

H1: There is a difference in wives' influence based on the products/services purchased.

ANOVA was used to compare the differences in wives' influence based on the products/services purchased. The descriptive statistics for this analysis is given in Table 3, the ANOVA in Table 4 and the post hoc analysis in Table 5.

The mean values in Table 3 indicate that when purchasing decisions are based on products alone, most of the products are purchased through joint decisions with cars $(M=1.62)$ and computers $(M=1.63)$ being the only products for which the decision is the husband's. For children's clothes, wife's clothes and groceries, purchasing decision is made by the wife. Table 4 indicates that there are significant differences 
IJMS 19 (2), 47-73 (2012)

in the wife's influence based on the products/services purchased $(\mathrm{p}<$ .001). Subsequent post hoc analysis shown in Table 5 shows that there are significant differences in the wife's influence pattern in almost all the products/services.

\section{Table 3}

Descriptive Statistics Based on Products/Services

\begin{tabular}{lcc}
\hline Products/Services & Mean & Std. deviation \\
\hline Furniture & 2.04 & 0.45 \\
Electrical & 1.85 & 0.50 \\
Computers & 1.63 & 0.56 \\
Children's clothes & 2.39 & 0.49 \\
Wife's clothes & 2.54 & 0.49 \\
Husband's clothes & 1.82 & 0.58 \\
Vacation & 1.87 & 0.39 \\
Eating out & 1.86 & 0.43 \\
Education & 1.95 & 0.51 \\
Entertainment & 1.92 & 0.51 \\
Bank accounts & 1.86 & 0.48 \\
Insurance & 1.70 & 0.55 \\
Home & 1.70 & 0.47 \\
Cars & 1.62 & 0.48 \\
Groceries & 2.40 & 0.53 \\
\hline
\end{tabular}

Table 4

ANOVA Result Based on Products/Services

\begin{tabular}{lcrrrr}
\hline & Sum of squares & \multicolumn{1}{c}{ df } & Mean square & F & Sig. \\
\hline Between groups & 1127.948 & 14 & 80.568 & 326.004 & .000 \\
Within groups & 3656.648 & 14796 & .247 & & \\
Total & 4784.596 & 14810 & & & \\
\hline
\end{tabular}

*the mean difference is significant at the 0.05 level. 
IJMS 19 (2), 47-73 (2012)

\section{Table 5}

Post Hoc Analysis Based on Products/Services

\begin{tabular}{|c|c|c|c|c|c|c|}
\hline \multirow[t]{2}{*}{ (I) Product type } & \multirow[t]{2}{*}{ (J) Product type } & \multirow{2}{*}{$\begin{array}{c}\text { Mean } \\
\text { difference } \\
(\mathrm{I}-\mathrm{J})\end{array}$} & \multirow[t]{2}{*}{$\begin{array}{l}\text { Std. } \\
\text { error }\end{array}$} & \multirow[t]{2}{*}{ Sig. } & \multicolumn{2}{|c|}{$\begin{array}{c}95 \% \text { Confidence } \\
\text { interval }\end{array}$} \\
\hline & & & & & $\begin{array}{l}\text { Lower } \\
\text { bound }\end{array}$ & $\begin{array}{l}\text { Upper } \\
\text { bound }\end{array}$ \\
\hline \multirow{14}{*}{ Furniture } & Electrical & $.18597^{*}$ & .02122 & .000 & .1119 & .2601 \\
\hline & Computers & $.40708^{*}$ & .02309 & .000 & .3264 & .4877 \\
\hline & Groceries & $-.36246^{*}$ & .02202 & .000 & -.4394 & -.2855 \\
\hline & Children's clothes & $-.35603^{*}$ & .02128 & .000 & -.4304 & -.2817 \\
\hline & Wife's clothes & $-.50120^{*}$ & .02118 & .000 & -.5752 & -.4272 \\
\hline & Husband's clothes & $.21952^{*}$ & .02324 & .000 & .1383 & .3007 \\
\hline & Vacation & $.16378^{*}$ & .01898 & .000 & .0975 & .2301 \\
\hline & Eating out & $.18091^{*}$ & .01964 & .000 & .1123 & .2495 \\
\hline & Education & $.08227^{*}$ & .02167 & .016 & .0066 & .1580 \\
\hline & Entertainment & $.11974^{*}$ & .02171 & .000 & .0439 & .1956 \\
\hline & Bank accounts & $.17486^{*}$ & .02099 & .000 & .1015 & .2482 \\
\hline & Insurance & $.33739^{*}$ & .02260 & .000 & .2585 & .4163 \\
\hline & Home (buy/rent) & $.33583^{*}$ & .02065 & .000 & .2637 & .4080 \\
\hline & Cars & $.41982^{*}$ & .02088 & .000 & .3469 & .4928 \\
\hline \multirow{8}{*}{ Electricals } & Computers & $.22111^{*}$ & .02399 & .000 & .1373 & .3049 \\
\hline & Groceries & $-.54843^{*}$ & .02296 & .000 & -.6286 & -.4682 \\
\hline & Children's clothes & $-.54200^{*}$ & .02225 & .000 & -.6197 & -.4643 \\
\hline & Wife's clothes & $-.68717^{*}$ & .02216 & .000 & -.7646 & -.6098 \\
\hline & Education & $-.10369^{*}$ & .02262 & .001 & -.1827 & -.0247 \\
\hline & Insurance & $.15142^{*}$ & .02351 & .000 & .0693 & .2336 \\
\hline & Home (buy/rent) & $.14986^{*}$ & .02165 & .000 & .0743 & .2255 \\
\hline & Cars & $.23385^{*}$ & .02187 & .000 & .1575 & .3102 \\
\hline \multirow{9}{*}{ Computers } & Groceries & $-.76954^{*}$ & .02470 & .000 & -.8558 & -.6833 \\
\hline & Children's clothes & $-.76311^{*}$ & .02405 & .000 & -.8471 & -.6791 \\
\hline & Wife's clothes & $-.90828^{*}$ & .02396 & .000 & -.9920 & -.8246 \\
\hline & Husband's clothes & $-.18756^{*}$ & .02580 & .000 & -.2777 & -.0975 \\
\hline & Vacation & $-.24330^{*}$ & .02203 & .000 & -.3203 & -.1663 \\
\hline & Eating Out & $-.22617^{*}$ & .02260 & .000 & -.3051 & -.1472 \\
\hline & Education & $-.32480^{*}$ & .02439 & .000 & -.4100 & -.2396 \\
\hline & Entertainment & $-.28733^{*}$ & .02443 & .000 & -.3727 & -.2020 \\
\hline & Bank accounts & $-.23221^{*}$ & .02379 & .000 & -.3153 & -.1491 \\
\hline
\end{tabular}


IJMS 19 (2), 47-73 (2012)

\begin{tabular}{|c|c|c|c|c|c|c|}
\hline \multirow[t]{2}{*}{ (I) Product type } & \multirow[t]{2}{*}{ (J) Product type } & \multirow{2}{*}{$\begin{array}{c}\text { Mean } \\
\text { difference } \\
(\mathrm{I}-\mathrm{J})\end{array}$} & \multirow[t]{2}{*}{$\begin{array}{l}\text { Std. } \\
\text { error }\end{array}$} & \multirow[t]{2}{*}{ Sig. } & \multicolumn{2}{|c|}{$\begin{array}{c}95 \% \text { Confidence } \\
\text { interval }\end{array}$} \\
\hline & & & & & $\begin{array}{l}\text { Lower } \\
\text { bound }\end{array}$ & $\begin{array}{l}\text { Upper } \\
\text { bound }\end{array}$ \\
\hline \multirow{10}{*}{ Groceries } & Wife's clothes & $-.13874^{*}$ & .02293 & .000 & -.2188 & -.0586 \\
\hline & Husband's clothes & $.58198^{*}$ & .02484 & .000 & .4952 & .6688 \\
\hline & Vacation & $.52624^{*}$ & .02091 & .000 & .4532 & .5993 \\
\hline & Eating Out & $.54338^{*}$ & .02151 & .000 & .4682 & .6185 \\
\hline & Education & $.44474^{*}$ & .02338 & .000 & .3631 & .5264 \\
\hline & Entertainment & $.48221^{*}$ & .02342 & .000 & .4004 & .5640 \\
\hline & Bank accounts & $.53733^{*}$ & .02275 & .000 & .4579 & .6168 \\
\hline & Insurance & $.69985^{*}$ & .02424 & .000 & .6152 & .7845 \\
\hline & Home (buy/rent) & $.69829^{*}$ & .02244 & .000 & .6199 & .7767 \\
\hline & Cars & $.78228^{*}$ & .02265 & .000 & .7032 & .8614 \\
\hline \multirow{10}{*}{$\begin{array}{l}\text { Children's } \\
\text { clothes }\end{array}$} & Wife's clothes & $-.14517^{*}$ & .02222 & .000 & -.2228 & -.0676 \\
\hline & Husband's clothes & $.57555^{*}$ & .02419 & .000 & .4910 & .6601 \\
\hline & Vacation & $.51981^{*}$ & .02013 & .000 & .4495 & .5901 \\
\hline & Eating out & $.53694^{*}$ & .02075 & .000 & .4645 & .6094 \\
\hline & Education & $.43831^{*}$ & .02268 & .000 & .3591 & .5175 \\
\hline & Entertainment & $.47578^{*}$ & .02273 & .000 & .3964 & .5552 \\
\hline & Bank accounts & $.53089^{*}$ & .02204 & .000 & .4539 & .6079 \\
\hline & Insurance & $.69342^{*}$ & .02357 & .000 & .6111 & .7758 \\
\hline & Home (buy/rent) & $.69186^{*}$ & .02171 & .000 & .6160 & .7677 \\
\hline & Cars & $.77585^{*}$ & .02194 & .000 & .6992 & .8525 \\
\hline \multirow{9}{*}{ Wife's clothes } & Husband's clothes & $.72072^{*}$ & .02411 & .000 & .6365 & .8049 \\
\hline & Vacation & $.66498^{*}$ & .02002 & .000 & .5950 & .7349 \\
\hline & Eating out & $.68211^{*}$ & .02065 & .000 & .6100 & .7543 \\
\hline & Education & $.58348^{*}$ & .02259 & .000 & .5046 & .6624 \\
\hline & Entertainment & $.62095^{*}$ & .02263 & .000 & .5419 & .7000 \\
\hline & Bank accounts & $.67607^{*}$ & .02194 & .000 & .5994 & .7527 \\
\hline & Insurance & $.83859^{*}$ & .02348 & .000 & .7566 & .9206 \\
\hline & Home (buy/rent) & $.83703^{*}$ & .02162 & .000 & .7615 & .9125 \\
\hline & Cars & $.92102^{*}$ & .02184 & .000 & .8447 & .9973 \\
\hline \multirow{5}{*}{$\begin{array}{l}\text { Husband's } \\
\text { clothes }\end{array}$} & Education & $-.13724^{*}$ & .02453 & .000 & -.2229 & -.0516 \\
\hline & Entertainment & $-.09977^{*}$ & .02457 & .005 & -.1856 & -.0139 \\
\hline & Insurance & $.11787^{*}$ & .02536 & .000 & .0293 & .2064 \\
\hline & Home (buy/rent) & $.11631^{*}$ & .02364 & .000 & .0338 & .1989 \\
\hline & Cars & $.20030^{*}$ & .02384 & .000 & .1170 & .2836 \\
\hline
\end{tabular}


IJMS 19 (2), 47-73 (2012)

\begin{tabular}{|c|c|c|c|c|c|c|}
\hline \multirow[t]{2}{*}{ (I) Product type } & \multirow[t]{2}{*}{ (J) Product type } & \multirow{2}{*}{$\begin{array}{c}\text { Mean } \\
\text { difference } \\
(\mathrm{I}-\mathrm{J})\end{array}$} & \multirow[t]{2}{*}{$\begin{array}{l}\text { Std. } \\
\text { error }\end{array}$} & \multirow[t]{2}{*}{ Sig. } & \multicolumn{2}{|c|}{$\begin{array}{l}95 \% \text { Confidence } \\
\text { interval }\end{array}$} \\
\hline & & & & & $\begin{array}{l}\text { Lower } \\
\text { bound }\end{array}$ & $\begin{array}{l}\text { Upper } \\
\text { bound }\end{array}$ \\
\hline \multirow{4}{*}{ Vacation } & Education & $-.08151^{*}$ & .02054 & .008 & -.1532 & -.0098 \\
\hline & Insurance & $.17361^{*}$ & .02151 & .000 & .0985 & .2488 \\
\hline & Home (buy/rent) & $.17205^{*}$ & .01946 & .000 & .1041 & .2400 \\
\hline & Cars & $.25604^{*}$ & .01970 & .000 & .1872 & .3249 \\
\hline \multirow{4}{*}{ Eating out } & Education & $-.09864^{*}$ & .02115 & .000 & -.1725 & -.0248 \\
\hline & Insurance & $.15648^{*}$ & .02210 & .000 & .0793 & .2337 \\
\hline & Home (buy/rent) & $.15492^{*}$ & .02010 & .000 & .0847 & .2251 \\
\hline & Cars & $.23891^{*}$ & .02034 & .000 & .1678 & .3100 \\
\hline \multirow{4}{*}{ Education } & Bank accounts & $.09259^{*}$ & .02241 & .004 & .0143 & .1709 \\
\hline & Insurance & $.25511^{*}$ & .02392 & .000 & .1716 & .3387 \\
\hline & Home (buy/rent) & $.25356^{*}$ & .02209 & .000 & .1764 & .3307 \\
\hline & Cars & $.33754^{*}$ & .02231 & .000 & .2596 & .4155 \\
\hline \multirow{3}{*}{ Entertainment } & Insurance & $.21764^{*}$ & .02396 & .000 & .1339 & .3013 \\
\hline & Home (buy/rent) & $.21608^{*}$ & .02213 & .000 & .1388 & .2934 \\
\hline & Cars & $.30007^{*}$ & .02235 & .000 & .2220 & .3781 \\
\hline \multirow{3}{*}{ Bank accounts } & Insurance & $.16253^{*}$ & .02331 & .000 & .0811 & .2440 \\
\hline & Home (buy/rent) & $.16097^{*}$ & .02143 & .000 & .0861 & .2358 \\
\hline & Cars & $.24496^{*}$ & .02165 & .000 & .1693 & .3206 \\
\hline Insurance & Cars & $.08243^{*}$ & .02321 & .040 & .0013 & .1635 \\
\hline Home (buy/rent) & Cars & $.08399^{*}$ & .02132 & .009 & .0095 & .1585 \\
\hline
\end{tabular}

*the mean difference is significant at the 0.05 level.

H2: Wife's influence in family purchase decision making varies with her income.

Table 6 displays the mean values of the influence pattern for each product/service based on the income level of the wife. ANOVA was used to test if the means of the products/services were different based on the wife's income level. The ANOVA result is shown in Table 7, and the post hoc analysis in Table 8. 
IJMS 19 (2), 47-73 (2012)

\section{Table 6}

Mean Values of Influence Pattern for Products/Services Based on Wife's Income Level

\begin{tabular}{lcccccc}
\hline \multirow{2}{*}{ Products/Services } & \multicolumn{5}{c}{ Monthly income level (wife) } \\
\cline { 2 - 7 } & No & Less than & RM1001- & RM5001- & RM10001- & More than \\
& income & RM1k & RM5000 & RM10000 & RM15000 & RM20000 \\
\hline Furniture & 1.97 & 2.05 & 2.07 & 2.08 & 2.31 & 2.25 \\
Electrical & 1.80 & 1.87 & 1.86 & 1.98 & 2.15 & 2.33 \\
Computers & $1.54^{*}$ & 1.71 & $1.64^{*}$ & 1.84 & 1.72 & $2.42^{* *}$ \\
Groceries & $2.35^{* *}$ & $2.43^{* *}$ & $2.42^{* *}$ & $2.45^{* *}$ & $2.51^{* *}$ & $2.50^{* *}$ \\
Children's clothes & $2.35^{* *}$ & $2.45^{* *}$ & $2.41^{* *}$ & $2.45^{* *}$ & $2.44^{* *}$ & $2.42^{* *}$ \\
Wife's clothes & $2.49^{* *}$ & $2.54^{* *}$ & $2.56^{* *}$ & $2.63^{* *}$ & $2.56^{* *}$ & 2.33 \\
Husband's clothes & 1.85 & 1.85 & 1.81 & 1.73 & 1.72 & $1.25^{*}$ \\
Vacation & 1.84 & 2.01 & 1.87 & 1.87 & 1.97 & $2.42^{* *}$ \\
Eating out & 1.81 & 1.88 & 1.87 & 1.94 & 1.92 & 2.17 \\
Education & 1.86 & 2.03 & 2.00 & 2.06 & 2.15 & $2.58^{* *}$ \\
Entertainment & 1.89 & 1.98 & 1.91 & 1.98 & 2.00 & 2.17 \\
Bank account & 1.73 & 1.93 & 1.94 & 1.96 & 1.97 & $2.42^{* *}$ \\
Insurance & $1.59^{*}$ & 1.77 & 1.76 & 1.77 & 1.91 & $2.50^{* *}$ \\
Home & $1.62^{*}$ & 1.76 & 1.72 & 1.84 & 1.95 & 2.08 \\
Cars & $1.56^{*}$ & $1.66^{*}$ & $1.62^{*}$ & 1.74 & 1.87 & 2.25 \\
\hline
\end{tabular}

* = husband's decision, ${ }^{* *}=$ wife's decision.

Table 7

ANOVA Result Based on Wife's Income Level

\begin{tabular}{|c|c|c|c|c|c|c|}
\hline & & $\begin{array}{l}\text { Sum of } \\
\text { squares }\end{array}$ & $\mathrm{df}$ & $\begin{array}{l}\text { Mean } \\
\text { square }\end{array}$ & $F$ & Sig. \\
\hline & Between groups & 3.622 & 5 & .724 & 3.598 & .003 \\
\hline \multirow[t]{3}{*}{ Furniture } & Within groups & 200.144 & 994 & .201 & & \\
\hline & Total & 203.767 & 999 & & & \\
\hline & Between groups & 4.580 & 5 & .916 & 3.780 & .002 \\
\hline \multirow[t]{3}{*}{ Electricals } & Within groups & 240.397 & 992 & .242 & & \\
\hline & Total & 244.977 & 997 & & & \\
\hline & Between groups & 9.931 & 5 & 1.986 & 6.450 & .000 \\
\hline \multirow[t]{2}{*}{ Computers } & Within groups & 294.413 & 956 & .308 & & \\
\hline & Total & 304.344 & 961 & & & \\
\hline
\end{tabular}

(continued) 
IJMS 19 (2), 47-73 (2012)

\begin{tabular}{|c|c|c|c|c|c|c|}
\hline & & $\begin{array}{l}\text { Sum of } \\
\text { squares }\end{array}$ & $\mathrm{df}$ & $\begin{array}{l}\text { Mean } \\
\text { square }\end{array}$ & $\mathrm{F}$ & Sig. \\
\hline \multirow{3}{*}{ Groceries } & Between groups & 1.880 & 5 & .376 & 1.343 & .244 \\
\hline & Within groups & 277.688 & 992 & .280 & & \\
\hline & Total & 279.568 & 997 & & & \\
\hline \multirow{3}{*}{$\begin{array}{l}\text { Children's } \\
\text { clothes }\end{array}$} & Between groups & 1.293 & 5 & .259 & 1.063 & .379 \\
\hline & Within groups & 236.153 & 971 & .243 & & \\
\hline & Total & 237.446 & 976 & & & \\
\hline \multirow{3}{*}{ Wife's clothes } & Between groups & 2.076 & 5 & .415 & 1.704 & .131 \\
\hline & Within groups & 242.019 & 993 & .244 & & \\
\hline & Total & 244.095 & 998 & & & \\
\hline \multirow{3}{*}{$\begin{array}{l}\text { Husband's } \\
\text { clothes }\end{array}$} & Between groups & 2.605 & 5 & .521 & 1.555 & .170 \\
\hline & Within groups & 332.661 & 993 & .335 & & \\
\hline & Total & 335.266 & 998 & & & \\
\hline \multirow{3}{*}{ Vacation } & Between groups & 3.250 & 5 & .650 & 4.293 & .001 \\
\hline & Within groups & 148.374 & 980 & .151 & & \\
\hline & Total & 151.624 & 985 & & & \\
\hline \multirow{3}{*}{ Eating out } & Between groups & 2.028 & 5 & .406 & 2.255 & .047 \\
\hline & Within groups & 178.027 & 990 & .180 & & \\
\hline & Total & 180.055 & 995 & & & \\
\hline \multirow{3}{*}{ Education } & Between groups & 7.929 & 5 & 1.586 & 6.163 & .000 \\
\hline & Within groups & 254.225 & 988 & .257 & & \\
\hline & Total & 262.155 & 993 & & & \\
\hline \multirow{3}{*}{ Entertainment } & Between groups & 1.404 & 5 & .281 & 1.073 & .374 \\
\hline & Within groups & 254.728 & 973 & .262 & & \\
\hline & Total & 256.132 & 978 & & & \\
\hline \multirow{3}{*}{ Bank account } & Between groups & 11.950 & 5 & 2.390 & 11.071 & .000 \\
\hline & Within groups & 205.939 & 954 & .216 & & \\
\hline & Total & 217.888 & 959 & & & \\
\hline \multirow{3}{*}{ Insurance } & Between groups & 9.922 & 5 & 1.984 & 6.865 & .000 \\
\hline & Within groups & 278.934 & 965 & .289 & & \\
\hline & Total & 288.856 & 970 & & & \\
\hline \multirow{3}{*}{ Home } & Between groups & 6.132 & 5 & 1.226 & 5.673 & .000 \\
\hline & Within groups & 213.817 & 989 & .216 & & \\
\hline & Total & 219.949 & 994 & & & \\
\hline \multirow{3}{*}{ Cars } & Between groups & 5.373 & 5 & 1.075 & 4.730 & .000 \\
\hline & Within groups & 225.153 & 991 & .227 & & \\
\hline & Total & 230.526 & 996 & & & \\
\hline
\end{tabular}

* The mean difference is significant at the 0.05 level. 
The data in Table 6 indicates that the influence of the wives tends to increase with income levels. Also noted are that few products tend to be wife-dominant at all income levels. Examples of these products are groceries and wife's clothes.

The ANOVA result in Table 7 shows that in the purchases of 10 products/services, there is a significant influence of income in determining wife's influence. These products/services are: furniture $(p<0.003)$, electrical $(p<0.002)$, computers $(p<0.001)$, vacation $(p=$ $0.001)$, eating out $(\mathrm{p}<0.047)$, education $(\mathrm{p}<0.001)$, bank account ( $\mathrm{p}$ $<0.000)$, insurance $(\mathrm{p}<0.001)$, homes $(\mathrm{p}<0.001)$ and cars $(\mathrm{p}<0.001)$. Post hoc analysis in Table 8 shows only the products/services for which there are significant differences based on wife's income level.

\section{Table 8}

Post Hoc Analysis Based the Income Level of the Wife

\begin{tabular}{|c|c|c|c|c|c|c|c|}
\hline \multirow[t]{2}{*}{$\begin{array}{l}\text { Dependent } \\
\text { variable }\end{array}$} & \multirow[t]{2}{*}{$\begin{array}{l}\text { (I) Monthly } \\
\text { income (wife) }\end{array}$} & \multirow[t]{2}{*}{$\begin{array}{l}\text { (J) Monthly income } \\
\text { (wife) }\end{array}$} & \multirow[t]{2}{*}{$\begin{array}{c}\text { Mean } \\
\text { difference } \\
(\mathrm{I}-\mathrm{J})\end{array}$} & \multirow[t]{2}{*}{$\begin{array}{l}\text { Std. } \\
\text { error }\end{array}$} & \multirow[t]{2}{*}{ Sig. } & \multicolumn{2}{|c|}{$\begin{array}{c}95 \% \\
\text { Confidence } \\
\text { interval }\end{array}$} \\
\hline & & & & & & $\begin{array}{l}\text { Lower } \\
\text { bound }\end{array}$ & $\begin{array}{l}\text { Upper } \\
\text { bound }\end{array}$ \\
\hline Furniture & No income & RM1001-RM5000 & $-.10384^{*}$ & .03157 & .013 & -.1940 & -.0137 \\
\hline Electrical & No income & RM5001-RM10000 & $-.17787^{*}$ & .05837 & .029 & -.3445 & -.0112 \\
\hline \multirow{3}{*}{ Computers } & \multirow{2}{*}{ No income } & RM5001-RM10000 & $-.30013^{*}$ & .06605 & .000 & -.4888 & -.1115 \\
\hline & & More than RM20000 & $-.87389^{*}$ & .27895 & .022 & -1.6704 & -.0773 \\
\hline & RM1001-RM5000 & RM5001-RM10000 & $-.20580^{*}$ & .06556 & .022 & -.3930 & -.0186 \\
\hline \multirow{3}{*}{ Vacation } & \multirow{2}{*}{ No income } & Less than RM1k & $-.16679^{*}$ & .04687 & .005 & -.3006 & -.0330 \\
\hline & & More than RM20000 & $-.57552^{*}$ & .19556 & .039 & -1.1339 & -.0171 \\
\hline & Less than RM1k & RM1001-RM5000 & $.14079^{*}$ & .04656 & .031 & .0078 & .2737 \\
\hline \multirow{3}{*}{ Education } & \multirow{3}{*}{ No income } & Less than RM1k & $-.17339^{*}$ & .06014 & .046 & -.3451 & -.0017 \\
\hline & & RM1001-RM5000 & $-.13872^{*}$ & .03577 & .002 & -.2409 & -.0366 \\
\hline & & RM5001-RM10000 & -.20156 & .06072 & .012 & -.3749 & -.0282 \\
\hline \multirow{4}{*}{$\begin{array}{l}\text { Bank } \\
\text { account }\end{array}$} & \multirow{4}{*}{ No income } & Less than RM1k & $-.20307^{*}$ & 05779 & .006 & -.3681 & -.0380 \\
\hline & & RM1001-RM5000 & $-.21465^{*}$ & .03325 & .000 & -.3096 & -.1197 \\
\hline & & RM5001-RM10000 & $-.23223^{*}$ & .05579 & .000 & -.3915 & -.0729 \\
\hline & & More than RM20000 & $-.68811^{*}$ & .23354 & .039 & -1.3550 & -.0212 \\
\hline
\end{tabular}

(continued) 


\begin{tabular}{|c|c|c|c|c|c|c|c|}
\hline \multirow[t]{2}{*}{$\begin{array}{l}\text { Dependent } \\
\text { variable }\end{array}$} & \multirow[t]{2}{*}{$\begin{array}{l}\text { (I) Monthly } \\
\text { income (wife) }\end{array}$} & \multirow[t]{2}{*}{$\begin{array}{l}\text { (J) Monthly income } \\
\text { (wife) }\end{array}$} & \multirow[t]{2}{*}{$\begin{array}{c}\text { Mean } \\
\text { difference } \\
(\mathrm{I}-\mathrm{J})\end{array}$} & \multirow[t]{2}{*}{$\begin{array}{l}\text { Std. } \\
\text { error }\end{array}$} & \multirow[t]{2}{*}{ Sig. } & \multicolumn{2}{|c|}{$\begin{array}{c}95 \% \\
\text { Confidence } \\
\text { interval }\end{array}$} \\
\hline & & & & & & $\begin{array}{l}\text { Lower } \\
\text { bound }\end{array}$ & $\begin{array}{l}\text { Upper } \\
\text { bound }\end{array}$ \\
\hline \multirow{2}{*}{ Insurance } & \multirow{2}{*}{ No income } & RM1001-RM5000 & $-.17038^{*}$ & .03832 & .000 & -.2798 & -.0610 \\
\hline & & More than RM20000 & $-.91012^{*}$ & .27022 & .010 & -1.6817 & -.1385 \\
\hline \multirow{2}{*}{ Home } & \multirow{2}{*}{ No income } & RM1001-RM5000 & $-.10316^{*}$ & .03279 & .021 & -.1968 & -.0095 \\
\hline & & RM5001-RM10000 & $-.22252^{*}$ & .05514 & .001 & -.3800 & -.0651 \\
\hline \multirow{2}{*}{ Cars } & \multirow{2}{*}{ No income } & RM5001-RM10000 & $-.18603^{*}$ & .05652 & .013 & -.3474 & -.0247 \\
\hline & & More than RM20000 & $-.69274^{*}$ & .23955 & .045 & -1.3767 & -.0088 \\
\hline
\end{tabular}

* The mean difference is significant at the 0.05 level.

For the purchase of furniture, even though it is a joint decision at all income levels of the wives, higher earning wives were relatively more influential as is hypothesized. Wives in the RM10,000 to RM15,000 inome bracket are relatively the most influential in terms of furniturepurchasing decisions. The post hoc analysis in Table 8 shows that for furniture, there were significant differences between the no income and the RM1000 to RM5000 income groups.

The findings also revealed that wives with higher earning power were relatively more influential and the purchase decision for electrical is a joint decision regardless of the income level of the wife. In the post hoc analysis for electrical appliance, only no income and RM5,000 to RM10,000 groups had significant differences as shown in Table 8.

For the purchase of computers, it was the husband's decision for the no income and the RM1001-RM5,000 groups but the wife's decision for those earning above RM20,000. It was a joint decision for the rest of the income groups. The post hoc analysis in Table 8 shows that there are significant differences between the no income group and the RM1,000-RM5,000 group and between the no income group and the above RM20,000 group.

With respect to vacation, the finding shows that it was a joint decision up to the RM15,000 group but was the wife's decision for those earning above RM20,000. The less than the RM1,000 income group was not only significantly different from the RM1,000 to RM5,000 group 
according to the post hoc analysis in Table 8 but was also relatively more influential. Otherwise higher income wives had relatively more influence. The post hoc analysis also shows that there were significant differences between the no income group and the less than RM1,000 and the more than RM20,000 groups.

As for the purchase of education, the finding shows that wives earning more than RM20,000 had autonomous buying decisions otherwise it was a joint decision. The influence pattern indicated an upward trend that followed the income earning levels of the wives, with a slight deviation for the less than RM1,000 group which seemed to have more influence than the RM1,000 to RM5,000 groups even though it was not significant according to the post hoc analysis in Table 8. The post hoc also shows that there were significant differences between the no income group and the less than RM1,000, RM1,000 to RM5,000 and RM5,000 to RM10,000 groups.

The trend in the finding indicates that the influence of the wife increases with respect to her earning power. For those earning more than RM20,000 the purchase decision for bank account was theirs, otherwise it has a joint decision for all the other income levels. The post hoc analysis in Table 8 also shows that there were significant differences between the no income group and the less than RM1,000, the RM1,000 to RM5,000, the RM5,000 to RM10,000, and the more than RM20,000 groups.

For the purchase of insurance, the finding shows that for the no income group, it was the husband's decision and for the more than RM20,000 group, it was the wife's decision; it was a joint decision for all the other income level groups. The trend also shows that the wife's influence increased according to her income earning level. The post hoc analysis in Table 8 also shows that there were significant differences between the no income group and the RM1,000 to RM5,000, and the more than RM20,000 groups.

As for the purchase of homes, it was the husband's decision for the no income group but a joint decision for all the other income groups. The wife's influence increased with earning power except for the less than RM1,000 income level group, which seemed to have relatively more influence than the RM1,000 to RM5,000 income group, even though it was not significant according to the post hoc analysis in Table 8. The post hoc analysis also shows that there were significant differences 
between the no income group and the RM1,000 to RM5,000 and the RM5,000, to RM10,000 groups.

With respect to the purchase decisions for cars, the finding shows that it was the husband's decision up to the RM5,000 income level but it was a joint decision for the rest of the income levels. Here again, the finding shows the wife's influence increased with earning power except for the less than RM1,000 income level group, which seemed to have relatively more influence than the RM1,000 to RM5,000 income group, even though it was not significant according to the post hoc analysis in Table 8 . The post hoc analysis also shows that there were significant differences between the no income group and the RM5,000 to RM10,000 and the more than RM20,000 groups.

\section{H2: Wives' influence in family purchase decision-making varies with wives' occupation.}

The same procedure used for the income level of the wife was used to test for differences based on the occupation of the wife. The mean values of the infuence patterns of wives for the products/services based on the wives' occupations are shown in Table 9. The ANOVA result based on the occupation of the wife is shown in Table 10 and the post hoc analysis in Table 11.

An examination of Table 9 reveals that there was no definite pattern of wives' influence based on the occupation of the wife. The mean values in Table 9 show that the purchase decision was husbanddominant in the following scenarios: when the wife was a student for electrical $(M=1.57)$, computers $(M=1.56)$, vacation $(M=1.67)$, bank account $(M=1.56)$, eating out $(M=1.57)$, insurance $(M=1.33)$, homes $(\mathrm{M}=1.33)$ and cars $(\mathrm{M}=1.29)$; when she was a housewife for computers $(M=1.53)$, insurance $(M=1.61)$, homes $(M=1.64)$ and cars $(M=1.56)$; when she is retired for insurance $(M=1.59)$ and cars $(\mathrm{M}=1.49)$; when she was in management for computers $(\mathrm{M}=$ $1.64)$, and cars $(M=1.63)$; when she was clerical $(M=1.66)$ and in entrepreneurship ( $M=1.67)$ for cars. In the case of professional, entrepreneur, housewife and other occupations, it was the wives' decision to buy groceries, children's and wife's clothes. It was also the wives' decision in the following cases: buying the wife's clothes when she was in management, clerical or retired; buying children's clothes when she was retired or a student; buying groceries when she was in clerical occupation. In all other cases, it was basically a joint decision for each product/service. 
IJMS 19 (2), 47-73 (2012)

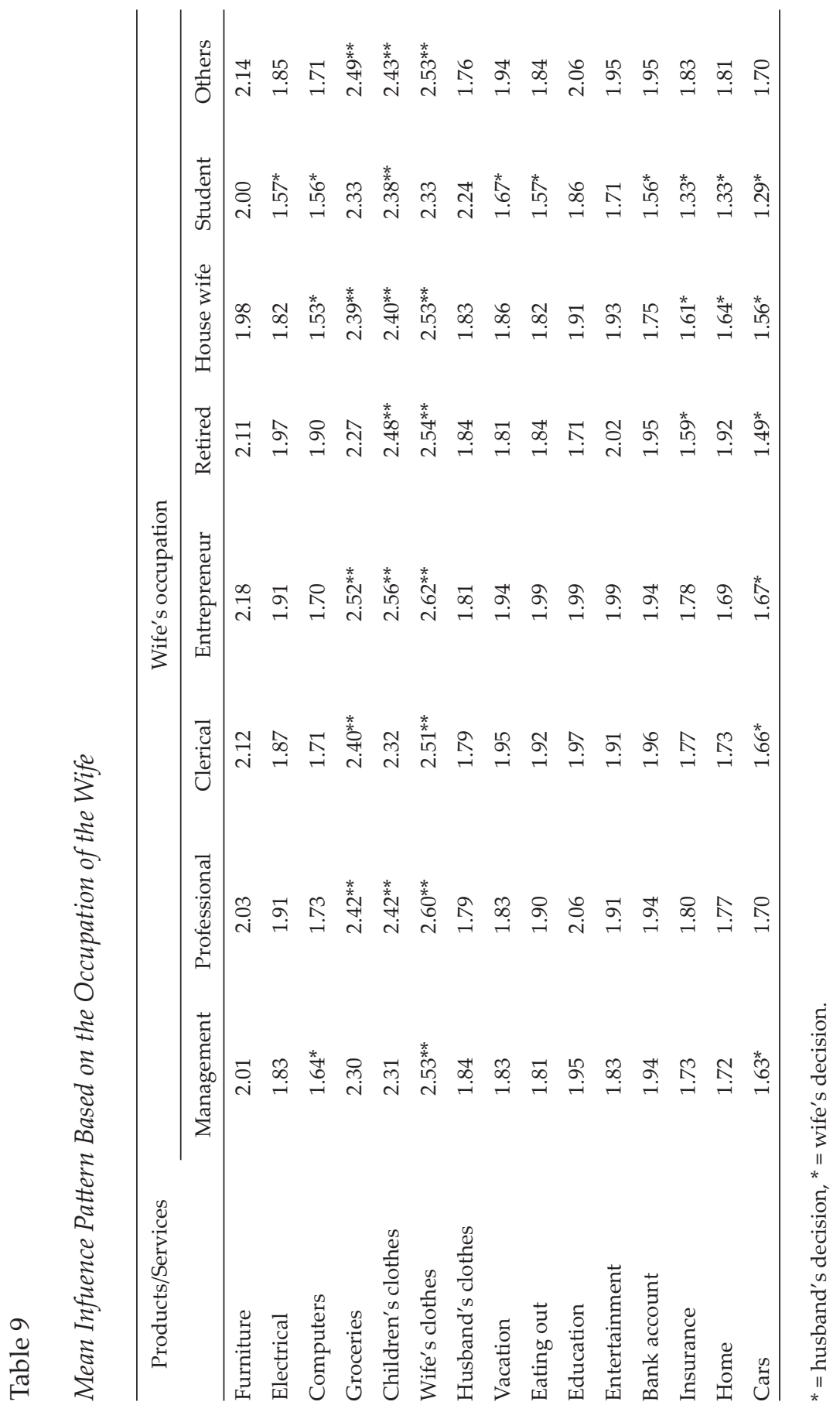


The ANOVA result in Table 10 shows that there were significant differences for furniture $(\mathrm{p}<0.001)$, computers $(\mathrm{p}<0.001)$, vacation $(\mathrm{p}<0.034)$, eating out $(\mathrm{p}<0.009)$, education $(\mathrm{p}<0.013)$, bank account $(\mathrm{p}<0.001)$, insurance $(\mathrm{p}<0.000)$, home $(\mathrm{p}<0.002)$ and cars $(\mathrm{p}<$ 0.008 ). The subsequent post hoc analysis is given in Table 11. The post hoc result in Table 11 indicates that for furniture, clerical and entrepreneur wives had significant differences with housewives; for bank account, management, professional and clerical wives had significant differenecs with housewives; for insurance, professional and clerical wives had significant differences with housewives; and for computers, professional and clerical wives had significant differences with housewives.

Table 10

ANOVA Result Based on Wife's Occupation

\begin{tabular}{|c|c|c|c|c|c|c|}
\hline & & $\begin{array}{l}\text { Sum of } \\
\text { squares }\end{array}$ & $\mathrm{df}$ & $\begin{array}{l}\text { Mean } \\
\text { square }\end{array}$ & $\mathrm{F}$ & Sig. \\
\hline \multirow{3}{*}{ Furniture } & Between groups & 4.864 & 7 & .695 & 3.463 & .001 \\
\hline & Within groups & 198.815 & 991 & .201 & & \\
\hline & Total & 203.679 & 998 & & & \\
\hline \multirow{3}{*}{ Electricals } & Between groups & 1.938 & 7 & .277 & 1.127 & .344 \\
\hline & Within groups & 243.016 & 989 & .246 & & \\
\hline & Total & 244.954 & 996 & & & \\
\hline \multirow{3}{*}{ Computers } & Between groups & 8.921 & 7 & 1.274 & 4.113 & .000 \\
\hline & Within groups & 295.286 & 953 & .310 & & \\
\hline & Total & 304.207 & 960 & & & \\
\hline \multirow{3}{*}{ Groceries } & Between groups & 3.089 & 7 & .441 & 1.581 & .137 \\
\hline & Within groups & 276.118 & 989 & .279 & & \\
\hline & Total & 279.207 & 996 & & & \\
\hline \multirow{3}{*}{ Children's clothes } & Between groups & 3.326 & 7 & .475 & 1.965 & .057 \\
\hline & Within groups & 234.045 & 968 & .242 & & \\
\hline & Total & 237.371 & 975 & & & \\
\hline \multirow{3}{*}{ Wife's clothes } & Between groups & 1.214 & 7 & .173 & .707 & .666 \\
\hline & Within groups & 242.668 & 990 & .245 & & \\
\hline & Total & 243.881 & 997 & & & \\
\hline \multirow{3}{*}{ Husband's clothes } & Between groups & 1.844 & 7 & .263 & .784 & .601 \\
\hline & Within groups & 332.754 & 990 & .336 & & \\
\hline & Total & 334.598 & 997 & & & \\
\hline
\end{tabular}

(continued) 
IJMS 19 (2), 47-73 (2012)

\begin{tabular}{|c|c|c|c|c|c|c|}
\hline & & $\begin{array}{l}\text { Sum of } \\
\text { squares }\end{array}$ & $\mathrm{df}$ & $\begin{array}{l}\text { Mean } \\
\text { square }\end{array}$ & $\mathrm{F}$ & Sig. \\
\hline \multirow{3}{*}{ Vacation } & Between groups & 2.329 & 7 & .333 & 2.181 & .034 \\
\hline & Within groups & 149.083 & 977 & .153 & & \\
\hline & Total & 151.412 & 984 & & & \\
\hline \multirow{3}{*}{ Eating out } & Between groups & 3.379 & 7 & .483 & 2.697 & .009 \\
\hline & Within groups & 176.655 & 987 & .179 & & \\
\hline & Total & 180.034 & 994 & & & \\
\hline \multirow{3}{*}{ Education } & Between groups & 4.682 & 7 & .669 & 2.564 & .013 \\
\hline & Within groups & 256.965 & 985 & .261 & & \\
\hline & Total & 261.647 & 992 & & & \\
\hline \multirow{3}{*}{ Entertainment } & Between groups & 1.812 & 7 & .259 & .987 & .439 \\
\hline & Within groups & 254.313 & 970 & .262 & & \\
\hline & Total & 256.125 & 977 & & & \\
\hline \multirow{3}{*}{ Bank account } & Between groups & 9.977 & 7 & 1.425 & 6.527 & .000 \\
\hline & Within groups & 207.688 & 951 & .218 & & \\
\hline & Total & 217.666 & 958 & & & \\
\hline \multirow{3}{*}{ Insurance } & Between groups & 8.639 & 7 & 1.234 & 4.243 & .000 \\
\hline & Within groups & 279.815 & 962 & .291 & & \\
\hline & Total & 288.454 & 969 & & & \\
\hline \multirow{3}{*}{ Home } & Between groups & 4.970 & 7 & .710 & 3.258 & .002 \\
\hline & Within groups & 214.890 & 986 & .218 & & \\
\hline & Total & 219.860 & 993 & & & \\
\hline \multirow{3}{*}{ Cars } & Between groups & 4.379 & 7 & .626 & 2.734 & .008 \\
\hline & Within groups & 226.000 & 988 & .229 & & \\
\hline & Total & 230.379 & 995 & & & \\
\hline
\end{tabular}

* The mean difference is significant at the 0.05 level.

Table 11

Post Hoc Analysis Based on Wife's Occupation

\begin{tabular}{|c|c|c|c|c|c|c|c|}
\hline \multirow[t]{2}{*}{$\begin{array}{l}\text { Dependent } \\
\text { variable }\end{array}$} & \multirow{2}{*}{$\begin{array}{c}\text { (I) } \\
\text { Occupation } \\
\text { (wife) }\end{array}$} & \multirow{2}{*}{$\begin{array}{c}(\mathrm{J}) \\
\begin{array}{c}\text { Occupation } \\
\text { (wife) }\end{array}\end{array}$} & \multirow{2}{*}{$\begin{array}{c}\text { Mean } \\
\text { difference } \\
(\mathrm{I}-\mathrm{J})\end{array}$} & \multirow[t]{2}{*}{$\begin{array}{l}\text { Std. } \\
\text { error }\end{array}$} & \multirow[t]{2}{*}{ Sig. } & \multicolumn{2}{|c|}{$\begin{array}{c}\text { 95\% Confidence } \\
\text { Interval }\end{array}$} \\
\hline & & & & & & $\begin{array}{l}\text { Lower } \\
\text { bound }\end{array}$ & $\begin{array}{l}\text { Upper } \\
\text { bound }\end{array}$ \\
\hline \multirow{2}{*}{ Furniture } & Clerical & Housewife & $.14643^{*}$ & .04183 & .011 & .0194 & .2735 \\
\hline & Entrepreneur & Housewife & $.20073^{*}$ & .06518 & .044 & .0028 & .3987 \\
\hline \multirow{3}{*}{$\begin{array}{c}\text { Bank } \\
\text { account }\end{array}$} & Management & Housewife & $.19404^{*}$ & .04900 & .002 & .0452 & .3429 \\
\hline & Professional & Housewife & $.19017^{*}$ & .04762 & .002 & .0455 & .3348 \\
\hline & Clerical & Housewife & $.21593^{*}$ & .04439 & .000 & .0811 & .3508 \\
\hline
\end{tabular}


IJMS 19 (2), 47-73 (2012)

\begin{tabular}{|c|c|c|c|c|c|c|c|}
\hline \multirow[t]{2}{*}{$\begin{array}{l}\text { Dependent } \\
\text { variable }\end{array}$} & \multirow{2}{*}{$\begin{array}{c}\text { (I) } \\
\text { Occupation } \\
\text { (wife) }\end{array}$} & \multirow{2}{*}{$\begin{array}{c}\text { (J) } \\
\text { Occupation } \\
\text { (wife) }\end{array}$} & \multirow{2}{*}{$\begin{array}{c}\text { Mean } \\
\text { difference } \\
(\mathrm{I}-\mathrm{J})\end{array}$} & \multirow[t]{2}{*}{$\begin{array}{l}\text { Std. } \\
\text { error }\end{array}$} & \multirow[t]{2}{*}{ Sig. } & \multicolumn{2}{|c|}{$\begin{array}{l}\text { 95\% Confidence } \\
\text { Interval }\end{array}$} \\
\hline & & & & & & & \\
\hline \multirow{2}{*}{ Insurance } & & & & 1 & .009 & .0293 & .3623 \\
\hline & Cleri & House & $.16266^{*}$ & .05133 & .034 & .0067 & .3186 \\
\hline \multirow{2}{*}{ Computers } & \multirow{2}{*}{ Housewife } & Professi & $-.20532^{*}$ & .05656 & .007 & -.3771 & -.0335 \\
\hline & & Clerical & $-.18110^{*}$ & .05277 & .014 & -.3414 & -.0208 \\
\hline
\end{tabular}

* The mean difference is significant at the 0.05 level.

H4: Wives' influence in family purchase decision-making varies with wives' education.

The mean values of the influence pattern based on the education level of the wife are shown in Table 12, ANOVA in Table 13 and the post hoc analysis in Table 14.

\section{Table 12}

Mean Infuence Pattern Based on the Wives' Education Level

\begin{tabular}{lcccccc}
\hline Products/Services & \multicolumn{7}{c}{ Wife's education level } \\
\cline { 2 - 7 } & $\begin{array}{c}\text { STPM or } \\
\text { equivalent }\end{array}$ & $\begin{array}{c}\text { Diploma or } \\
\text { equivalent }\end{array}$ & Others & $\begin{array}{c}\text { SPM or } \\
\text { equivalent }\end{array}$ & $\begin{array}{c}\text { Bachelor } \\
\text { degree or } \\
\text { equivalent }\end{array}$ & $\begin{array}{c}\text { Masters } \\
\text { or PhD }\end{array}$ \\
\hline Furniture & 2.08 & 2.05 & 2.04 & 2.01 & 2.04 & 2.13 \\
Electrical & 1.91 & 1.81 & 1.86 & 1.84 & 1.88 & 1.96 \\
Computers & $1.59^{*}$ & $1.61^{*}$ & $1.66^{*}$ & $1.66^{*}$ & 1.84 & $1.64^{*}$ \\
Groceries & 2.31 & $2.34^{* *}$ & $2.37^{* *}$ & $2.42^{* *}$ & $2.46^{* *}$ & $2.48^{* *}$ \\
Children's clothes & 2.26 & $2.35^{* *}$ & $2.39^{* *}$ & $2.40^{* *}$ & $2.48^{* *}$ & $2.50^{* *}$ \\
Wife's clothes & $2.44^{* *}$ & $2.50^{* *}$ & $2.44^{* *}$ & $2.56^{* *}$ & $2.63^{* *}$ & $2.64^{* *}$ \\
Husband's clothes & 1.80 & 1.84 & 1.66 & 1.85 & 1.75 & 2.00 \\
Vacation & 1.85 & 1.85 & 1.89 & 1.91 & 1.83 & 1.90 \\
Eating out & 1.86 & 1.86 & 1.73 & 1.86 & 1.86 & 2.01 \\
Education & 1.81 & 1.89 & 1.80 & 1.99 & 2.06 & 2.18 \\
Entertainment & 1.79 & 1.87 & 1.87 & 1.97 & 1.93 & 2.06 \\
Bank account & 1.86 & 1.86 & 1.79 & 1.84 & 1.94 & 1.93 \\
Insurance & 1.61 & 1.70 & 1.65 & 1.69 & 1.77 & 1.79 \\
Home & 1.76 & 1.66 & 1.70 & 1.68 & 1.73 & 1.90 \\
Cars & 1.64 & 1.59 & 1.52 & 1.61 & 1.66 & 1.81 \\
\hline
\end{tabular}

${ }^{*}=$ husband's decision, ${ }^{* *}=$ wife's decision. 
The mean values in Table 12 show that it was mostly a joint decision for buying most of the products/services for the different education levels of the wife. Purchase decision for wife's clothes is the wife's decision for all education levels. Similarly, purchase decisions for groceries and children's clothes were the wife's decision for all education levels except for the STPM or equivalent group where it became a joint decision. Buying decisions for computers and cars were the husband's except for wives with $\mathrm{PhD}$ or Masters where it was a joint decision. In addition, it was the husband's decision in the following scenarios: buying homes in the case of wives with diplomas or equivalent; buying insurance in the case of wives with STPM or equivalent and other qualifications; buying husband's clothes in the case of wives with other qualifications. The values in Table 12 also show that wives with $\mathrm{PhD}$ or Masters were relatively more influential for all the products/services except for computers and bank account where wives with Bachelor or equivalent degrees were more influential. Generally, wives with STPM or equivalent qualifications were relatively the least influential.

The ANOVA results in Table 13 indicate that there were significant differences in the influence pattern based on the education level of the wives for the following products/services: children's clothes $(p<0.010)$, wife's clothes $(p<0.007)$, husband's clothes $(p<0.029)$, education $(\mathrm{p}<0.001)$ and entertainment $(\mathrm{p}<0.009)$.

Table 13

ANOVA Analysis Based on the Education Level of the Wives

\begin{tabular}{llrrrrr}
\hline & & Sum of squares & \multicolumn{1}{c}{ df } & Mean square & F & \multicolumn{1}{c}{ Sig. } \\
\hline Furniture & Between groups & .843 & 5 & .169 & .824 & .532 \\
& Within groups & 202.250 & 989 & .204 & & \\
& Total & 203.093 & 994 & & & \\
Electricals & Between groups & 1.235 & 5 & .247 & 1.002 & .415 \\
& Within groups & 243.396 & 987 & .247 & & \\
& Total & 244.631 & 992 & & & \\
Computers & Between groups & 2.530 & 5 & .506 & 1.596 & .158 \\
& Within groups & 301.535 & 951 & .317 & & \\
& Total & 304.065 & 956 & & & \\
& Between groups & 2.466 & 5 & .493 & 1.762 & .118 \\
& Within groups & 276.259 & 987 & .280 & & \\
& Total & 278.724 & 992 & & & \\
\hline
\end{tabular}

(continued) 
IJMS 19 (2), 47-73 (2012)

\begin{tabular}{|c|c|c|c|c|c|c|}
\hline & & Sum of squares & $\mathrm{df}$ & Mean square & $\mathrm{F}$ & Sig. \\
\hline \multirow{3}{*}{$\begin{array}{l}\text { Children's } \\
\text { clothes }\end{array}$} & Between groups & 3.684 & 5 & .737 & 3.052 & .010 \\
\hline & Within groups & 233.220 & 966 & .241 & & \\
\hline & Total & 236.904 & 971 & & & \\
\hline \multirow[t]{3}{*}{ Wife's clothes } & Between groups & 3.865 & 5 & .773 & 3.192 & .007 \\
\hline & Within groups & 239.301 & 988 & .242 & & \\
\hline & Total & 243.166 & 993 & & & \\
\hline \multirow{3}{*}{$\begin{array}{l}\text { Husband's } \\
\text { clothes }\end{array}$} & Between groups & 4.178 & 5 & .836 & 2.500 & .029 \\
\hline & Within groups & 330.307 & 988 & .334 & & \\
\hline & Total & 334.486 & 993 & & & \\
\hline \multirow[t]{3}{*}{ Vacation } & Between groups & 1.125 & 5 & .225 & 1.461 & .200 \\
\hline & Within groups & 150.143 & 975 & .154 & & \\
\hline & Total & 151.268 & 980 & & & \\
\hline \multirow[t]{3}{*}{ Eating out } & Between groups & 1.818 & 5 & .364 & 2.011 & .075 \\
\hline & Within groups & 178.088 & 985 & .181 & & \\
\hline & Total & 179.906 & 990 & & & \\
\hline \multirow{3}{*}{ Education } & Between groups & 8.320 & 5 & 1.664 & 6.477 & .000 \\
\hline & Within groups & 252.530 & 983 & .257 & & \\
\hline & Total & 260.850 & 988 & & & \\
\hline \multirow{3}{*}{ Entertainment } & Between groups & 4.022 & 5 & .804 & 3.110 & .009 \\
\hline & Within groups & 250.348 & 968 & .259 & & \\
\hline & Total & 254.370 & 973 & & & \\
\hline \multirow{3}{*}{ Bank account } & Between groups & 1.847 & 5 & .369 & 1.624 & .151 \\
\hline & Within groups & 215.908 & 949 & .228 & & \\
\hline & Total & 217.755 & 954 & & & \\
\hline \multirow[t]{3}{*}{ Insurance } & Between groups & 2.118 & 5 & .424 & 1.420 & .214 \\
\hline & Within groups & 286.374 & 960 & .298 & & \\
\hline & Total & 288.492 & 965 & & & \\
\hline \multirow[t]{3}{*}{ Home } & Between groups & 2.175 & 5 & .435 & 1.969 & .081 \\
\hline & Within groups & 217.412 & 984 & .221 & & \\
\hline & Total & 219.587 & 989 & & & \\
\hline \multirow[t]{3}{*}{ Cars } & Between groups & 2.176 & 5 & .435 & 1.885 & .094 \\
\hline & Within groups & 227.593 & 986 & .231 & & \\
\hline & Total & 229.769 & 991 & & & \\
\hline
\end{tabular}

* The mean difference is significant at the 0.05 level.

The general finding shows that the influence pattern of wives increased with their education level. Purchasing decision for children's clothes was a joint decision for wives with STPM or equivalent qualifications but it was the wife's decision for all the other educational qualifications of the wives. The post hoc analysis in Table 14 indicates that STPM or equivalent holders had significant differences with bachelor degree or its equivalent holders. 
IJMS 19 (2), 47-73 (2012)

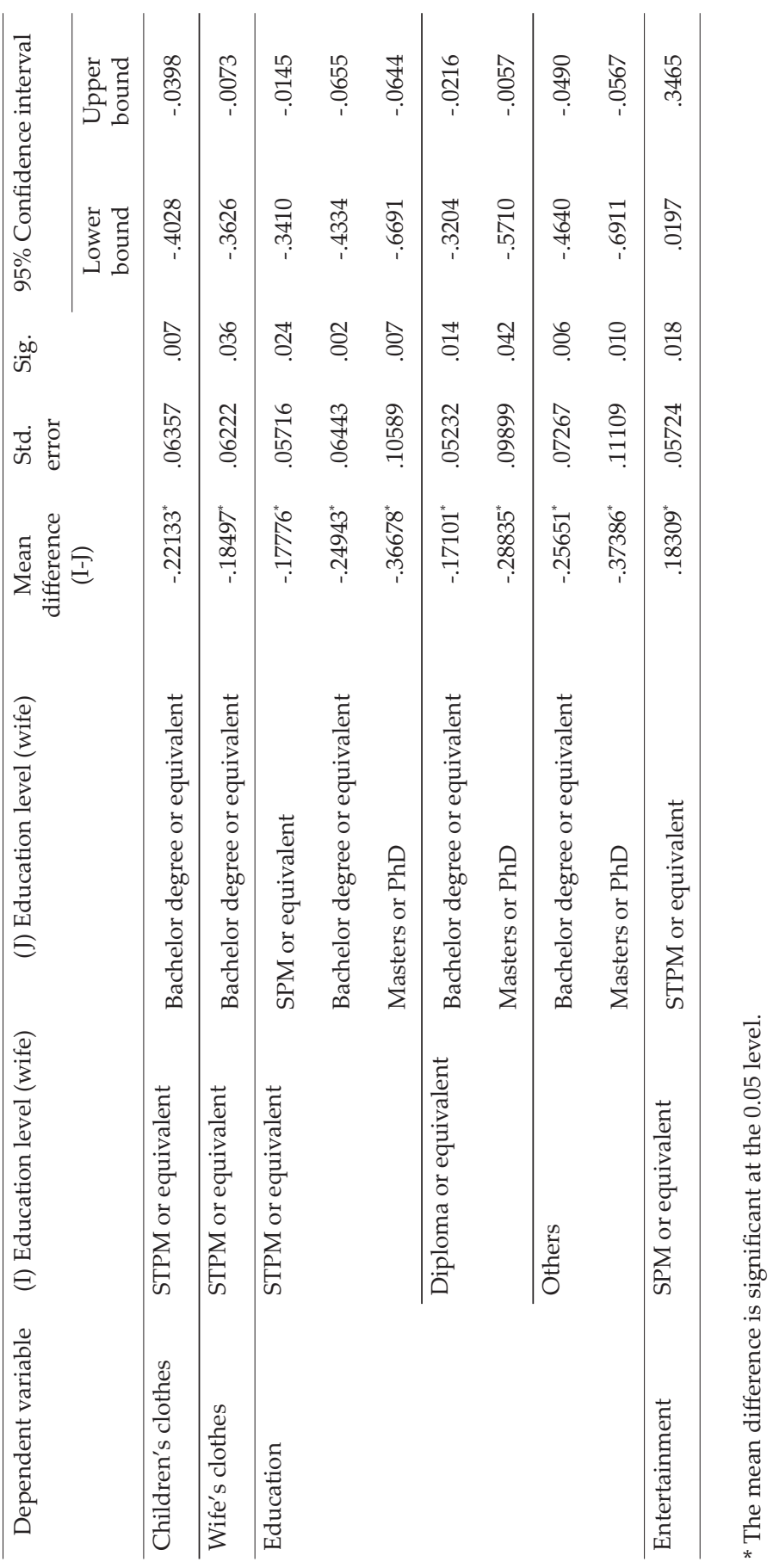


With regards to buying decisions for the wife's clothes, the finding indicates that it is the wife's decision at all levels of the wife's educational qualifications. Wives with masters or $\mathrm{PhD}$ 's are relatively more influential whilst those with STPM or equivalent and other qualifications were the least influential. The post hoc analysis in Table 14 shows that there were significant differences between wives with STPM or equivalent and wives with bachelor degrees or their equivalent qualifications.

As for the purchase of husband's clothes, it was found to be mostly a joint decision except for wives with other qualifications where it was the husband's decision. Here again wives with $\mathrm{PhD}$ or Masters degrees had the most influence. The post hoc analysis did not reveal any significant differences among the education levels of the wife.

The purchase decision for education was a joint decision across all educational qualifications of the wife. Masters or PhD holders were relatively more influential whilst STPM or its equivalent and other qualification holders had the least influence with respect to purchase decisions for education. The post hoc result in Table 14 shows that wives with STPM or equivalent qualifications had significant differences with SPM or its equivalent, bachelor or its equivalent and Masters or PhD holders. Similarly wives with diploma or equivalent qualifications had significant differences with bachelor or its equivalent and Masters or PhD holders. Also wives with other qualifications had significant differences with bachelor or its equivalent and Masters or PhD holders.

The finding indicates that for all levels of the wife's education, the entertainment buying decision was a joint one. Again, wives with STPM or equivalent qualifications were relatively the least influential whilst Masters and PhD holders were the most influential in terms buying decisions for entertainment. The post analysis shows that there were differences between SPM or its equivalent holders and STPM or its equivalent holders.

\section{Discussions and Conclusions}

The study aims to investigate family purchase decision-making in urban Malaysia and the factors affecting the decision-making process. Besides analysing the decision-making process, wife's resources (income, occupation and education) were investigated to determine their effects on family-purchase decisions. 
The findings of this study revealed that the majority of products/ services purchased for the family is a joint decision. For those very specific items such as computers, groceries and clothing, the findings indicated that they were bought jointly by the husbands and wives. It is interesting to note that the wife's influence generally decreased in the outcome stage, i.e., when products were actually purchased. The husband's bigger influence at this stage may be an actual manifestation of power in the family. This power is actually financial in nature, and would happen in a majority of the households where husbands earned more income. The findings revealed that wife's resources do positively affect her influence in purchase decisionmaking as it was shown that wives with higher income and education and better positions in their occupations had more power in their family purchase decision-making.

These findings help to shed some light for marketing practitioners in developing their marketing strategies for products used by the family. Marketers should take into consideration that many purchases for family consumption are done jointly. Thus, the target market should be identified with caution, as both husbands and wives are involved in the decision-making process. It is also noted from the findings that role structure is product specific and varies with the stages of the decision-making process. Even though the husbands have the ultimate purchase influence, the wives contribute in the earlier stage of the decision-making process.

Marketers should also take into consideration the changes in family values brought about by economic and social developments. Wives with more resources are relatively more exposed in the market, are open-minded, and consequently, take active roles in purchase decisions, especially those high-involvement products.

The findings in this study should be interpreted with caution as this study has several limitations. The sample of wives was taken from an urban setting, and may not be reflective of Malaysia in general. Also, the responses were taken only from the wife's perceptions. In the future, studies should also include the husband's perspectives to be less biased. Other psychographic variables and values can also be studied to complement the findings based on demographic variables. The study should also include more parts of Malaysia, and include the non-urban settings. Qualitative studies should also be conducted to examine the issues in family purchase decision-making from different 
perspectives. In conclusion, this study has found husbands, who hold the 'financial' power in the family, to be powerful in the purchases (outcome) made by the family. The influence of wives was found to vary by products and services, as well as their personal resources.

\section{References}

Hawkins, D. B., \& Coney, K. A. (2004). Consumer behaviour: Building marketing strategy (9th ed.). Boston: McGraw Hill.

Samsinar, M. S., \& Mary, A. (1996). Wives' involvement and the effects of sex role orientation in family decision-making. Proceedings of Multicultural Marketing Conference, Virginia, USA, 102-106.

Samsinar, M. S., Wong, F. Y., Dahlia, Z., Ruhana, B., \& Zalfa Laili H. (2004). The effects of spousal resources on family purchase decision-making in Malaysia: A replication. Malaysian Journal of Consumer and Family Economics, 7, 131-138.

Samsinar M. S., \& Rao, C. P. (2005). Family decision-making processes. Journal of Marketing and Communication, 1, 76-87.

Schiffman, L. G., \& Kanuk, L. L. (2010). Consumer behaviour. Upper Saddle River, New Jersey, USA: Pearson Education.

Xia, Y., Ahmed, Z. U., Hwa, N. K., Tan, W. L., \& Teo, W. (2006). Spousal influence in Singaporean family purchase decisionmaking process: A cross-cultural comparison. Asia Pacific Journal of Marketing and Logistics, 18, 201-222. 
